Iranian Journal of Forensic Medicine - ISSN 2383-0034

\title{
CT Scan and MRI Findings in Patients with Dementia
}

\author{
Reza Hajmanouchehri \\ Neurologist and Expert of the General Directorate of Medical Commission of the Legal Medicine Organization , \\ Tehran, Iran
}

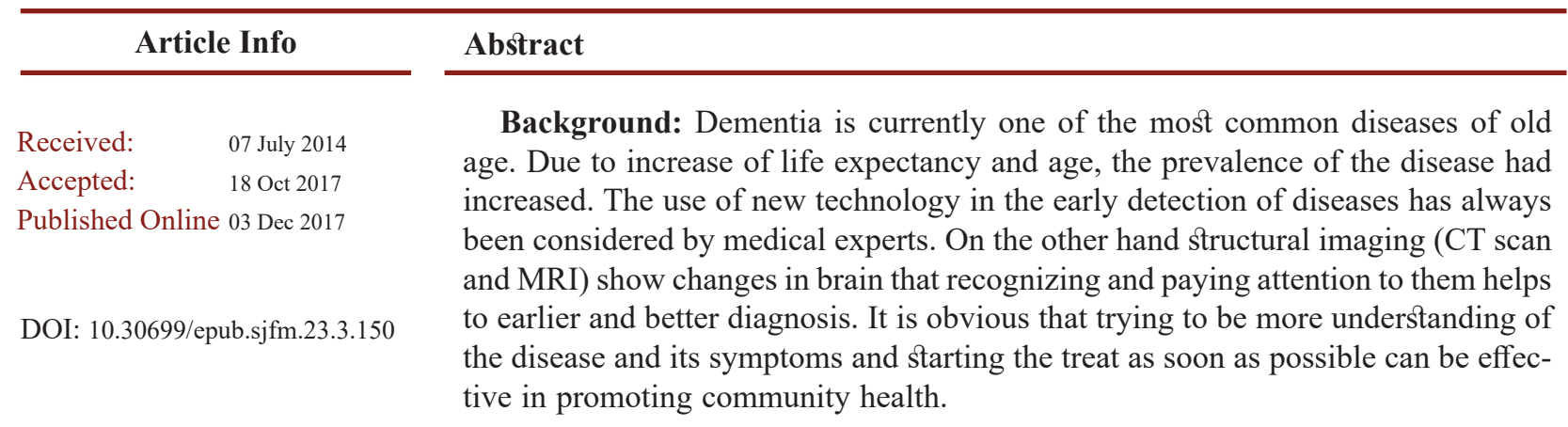

Review Article

Materials and Methods: With referring to databases PUBMED, MEDLINE and other authoritative, relevant articles and new articles were searched and then evaluated. Information was collected and presented.

Findings and Conclusion: Although the role of imaging in the diagnosis of dementia is not fundamental, but many changes occur in brain of patients with dementia can be detected in imaging. Some of these lesions, are created even before clinical symptoms; their detection in early diagnosis of disease can be helpful.

Keywords: Dementia, CT Scan, MRI

Copyright (C 2017, Ir J Forensic Med. This is an open-access article distributed under the terms of the Creative Commons Attribution-noncommercial 4.0 International License which permits copy and redistribute the material just in noncommercial usages, provided the original work is properly cited.

How to Cite This Article:

Hajmanouchehri R. CT Scan and MRI Findings in Patients with Dementia. Ir J Forensic. Med. 2017; 23 (3):150-159 


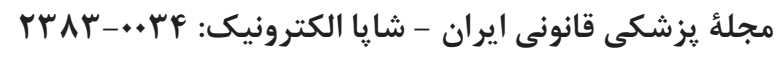

مقاله مرورى

يافته هاى CT اسكن و MRI در بيماران مبتلا به دمانس

رضا حاجمنوجهرى

نورولوزيست و كارشناس ادارة كل كميسيونهاى يزشكى سازمان بزشكى قانونى، تهران، ايران

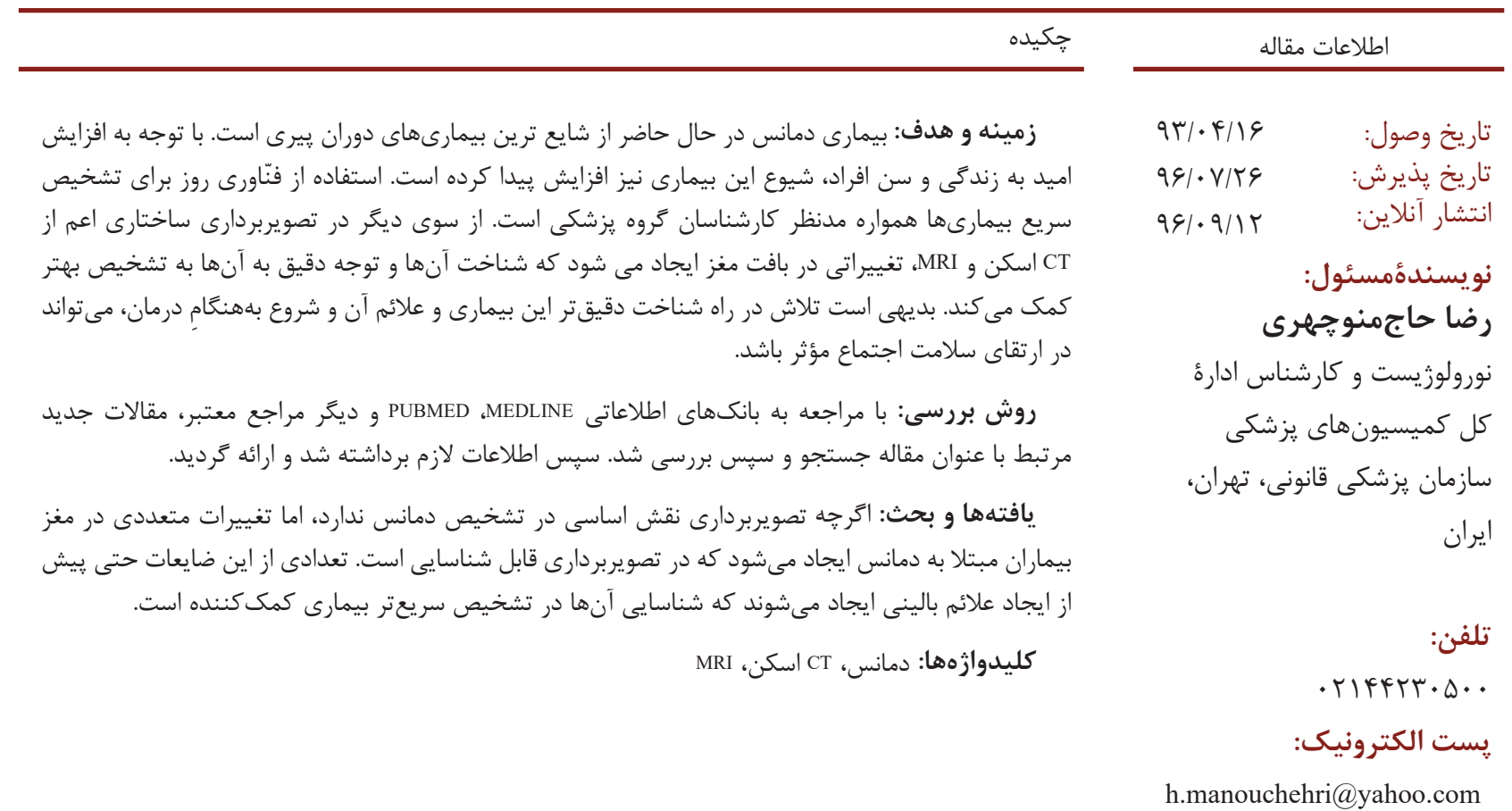

مقدمه

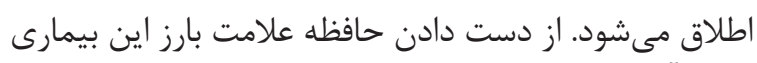

است. آلزايمر شايعترين نوع زوال عقل است (r (Y).

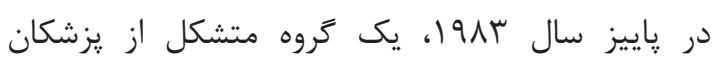

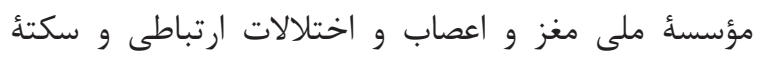

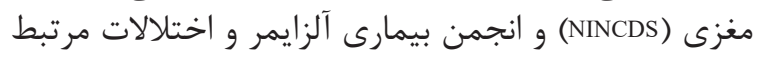

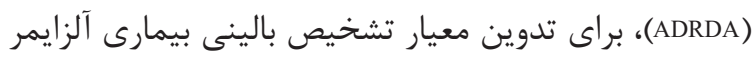

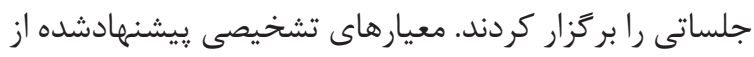

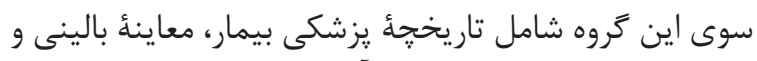

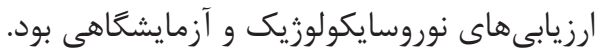

بيمارى آلزايمر براى نخستين بار در سال V• 19 آتوسط

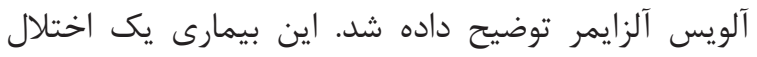

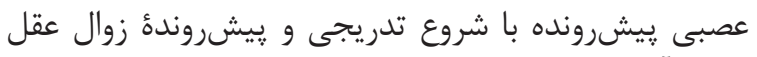

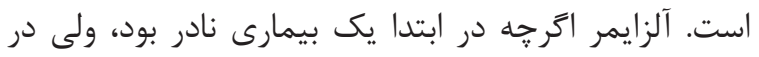

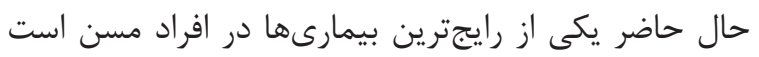

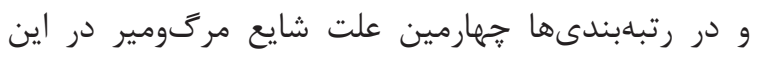

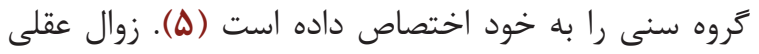

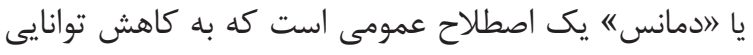

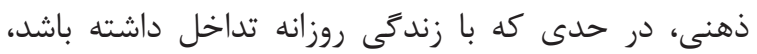


زمانى بيشنهاد مىشود كه ديكر روشها در تشخيص موفق نباشند.

اختلال شناختى و يا رفتارى دستِكم در دو حوزه از

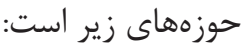

1- اختلال در حفظ و يادآورى اطلاعات جديد،

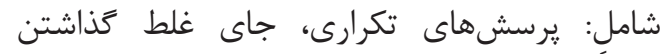

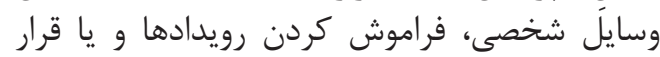

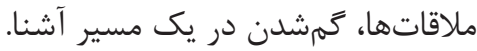

ץ- ناتوانى در انجام كارهاى يِيجيده و قضاوت ضعيف

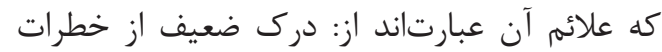

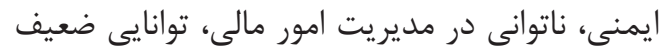

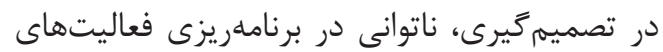

بيجيده.

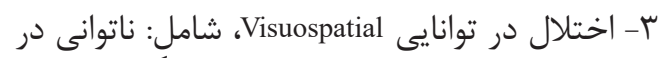

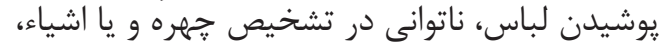
با وجود داشتن ديد نرمال. ب اختلال تكلم

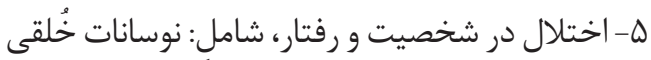

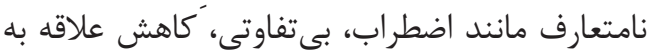

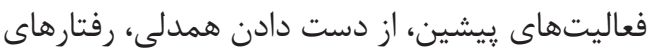

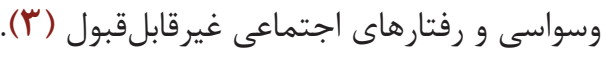

همجنين معيارهاى تشخيصى بيمارى آلزايمر به اين شكل

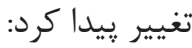

الف- آلز - إيمر محتمل

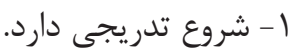

r-َّزارشى دقيق از زمان بدتر شدن اوضاع شدرد شناختى بيمار

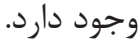

r-اختلال شناختى بهصورت اوليه و برجسته در شرححال و معاينه با يكى از تظاهرات زير بروز مئل اوليه وكند:

الف- فراموشى علامت غالب است:

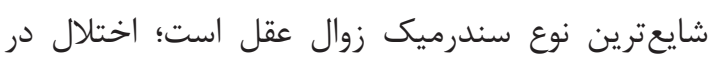

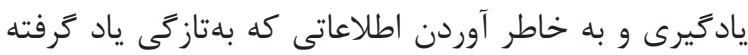

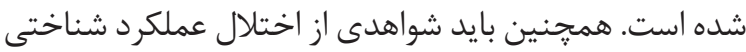

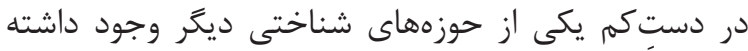
باشد.

\section{ب- غلبه با علائمى غير از فراموشى:}

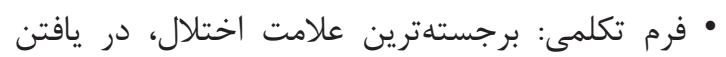

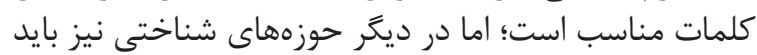
نقص وجود داشته باشد. • علائم Visuospatial: اشكال برجسته در شناخت فضايى،

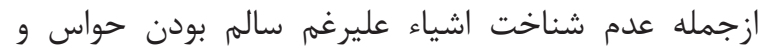

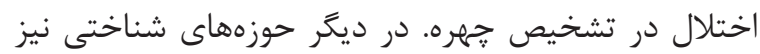
بايد نقص وجود داشته باشد.

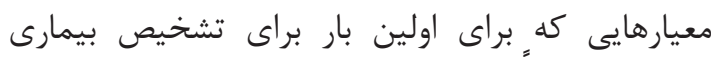

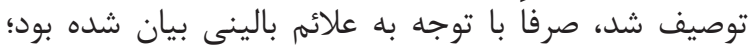

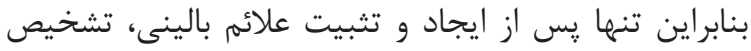

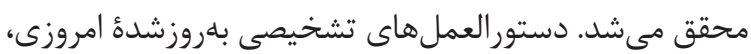

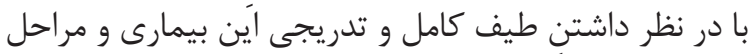

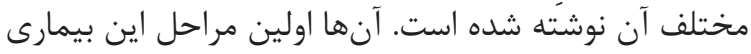

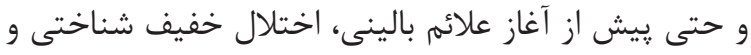

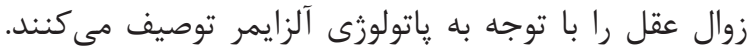

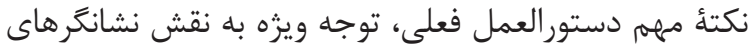

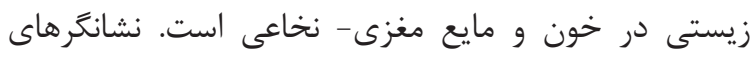

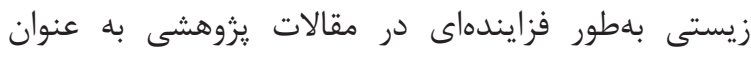

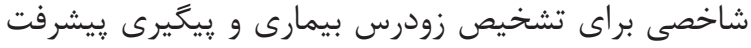

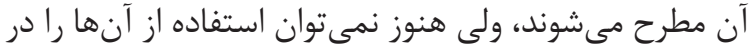

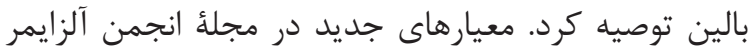

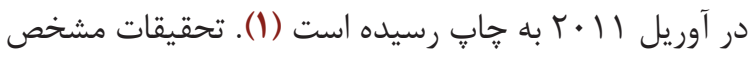

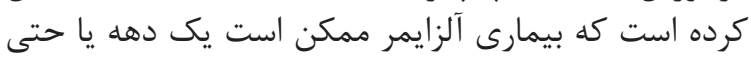

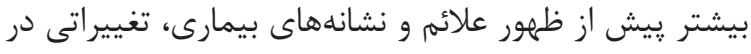

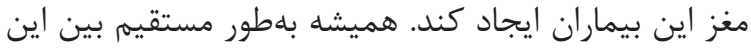

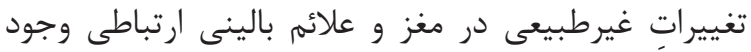

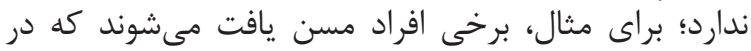

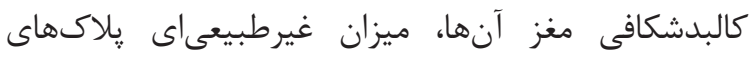

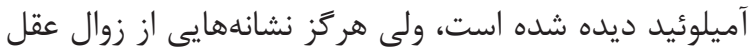

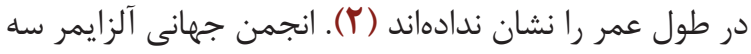
مرحله براى بيمارى توصيف كرده است:

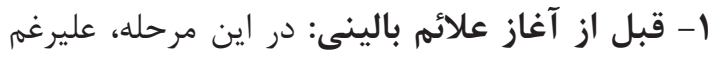

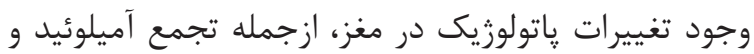

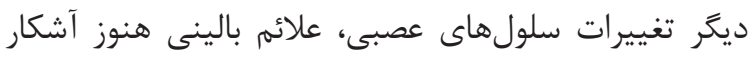

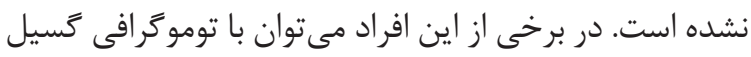

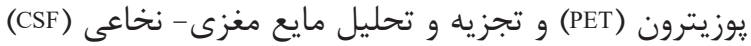

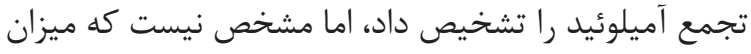

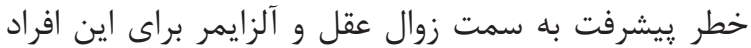
جه ميزان است.

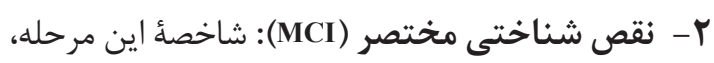

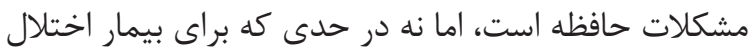
عملكرد ايجاد كند. اين افراد ممكن است به به آلزايمر دجار اختار

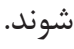

r- دمانس: زمانى تشخيص مطرح مىشود كه اختلالات

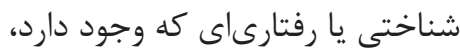
الف- با توانايى عملكرد فرد در محل كار يا فعاليتهاي دارئ معمول وى تداخل داشته باشد؛ ب- نسبت به سطح قبلى عملكرد خود افت داشته باشد؛

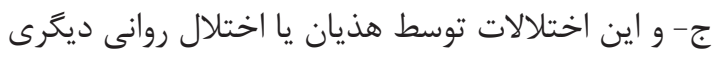

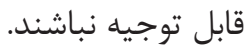

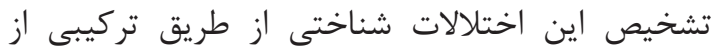

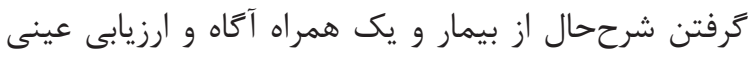

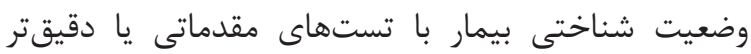

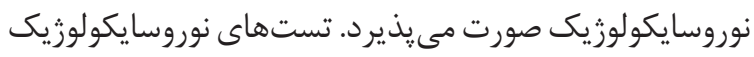


و سى ستىاسكن (CT) ممكن است علل غيردزنراتيو و (MRI)

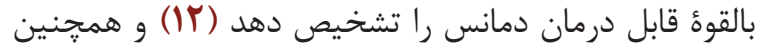

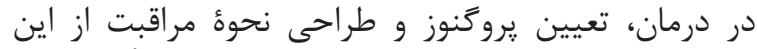

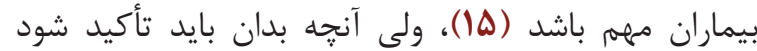

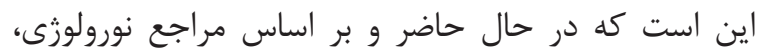

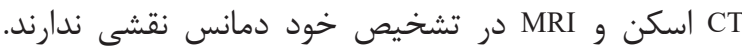

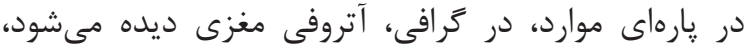

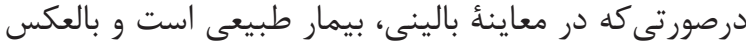

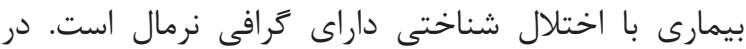

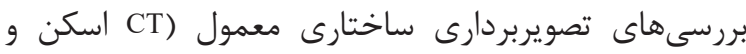

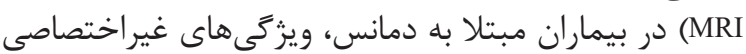

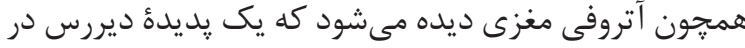

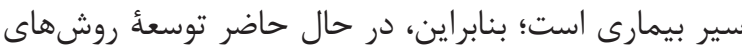

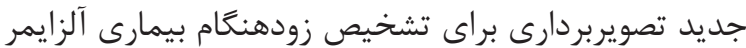

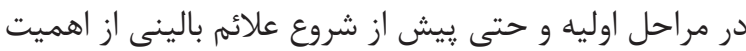

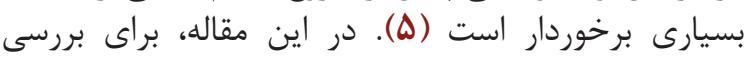

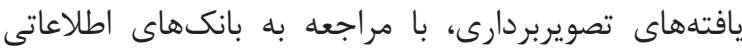
SUBMED ،MEDLINE

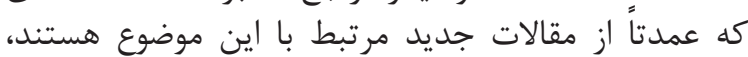

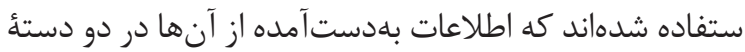

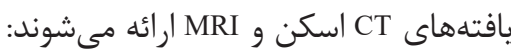

\section{الف- تغييرات مشاهدهده در CT اسكن:}

با افزايش سن، روند تحليل سلولها، همزمان با ديخر

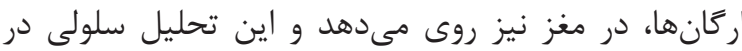

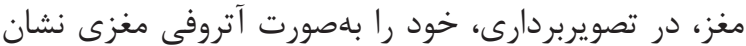

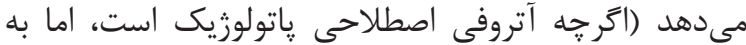

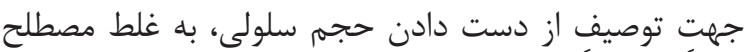

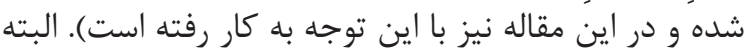

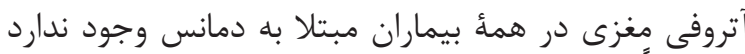

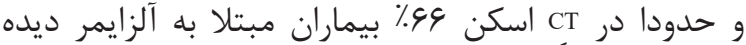

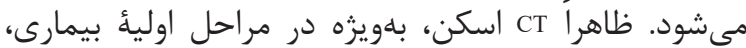

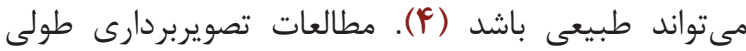

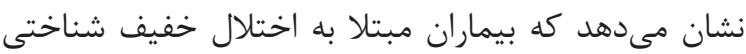

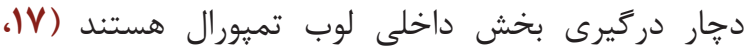

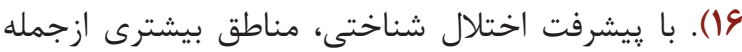

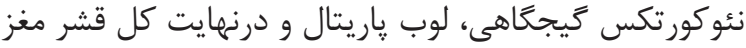

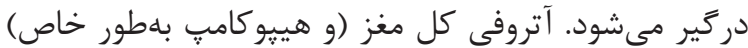

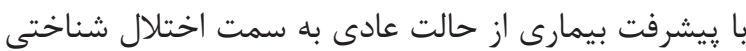

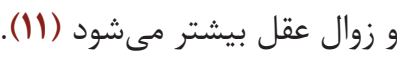

يافتهاى CT اسكن كه مؤيد وقوع دمانس هستند،

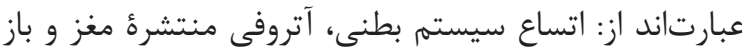

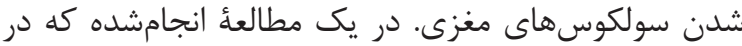

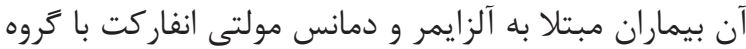

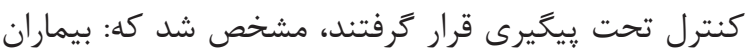

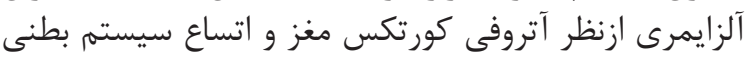

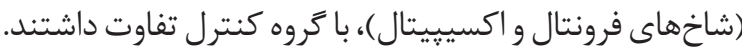

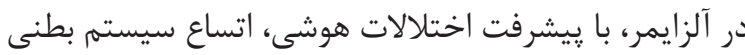

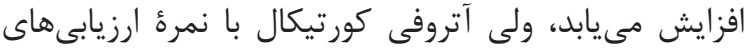

• اختلال عملكرد اجر ايى: برجستهترين نقص در استدلال،

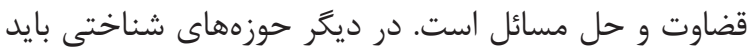
نقص وجود داشته باشد.

\section{ب- آلزايمر ممكن} 1 - - بيمارى سير غيرطبيعى دارد.

r-علائم اصلى و تشخيصى آلزايمر موجود است، اما

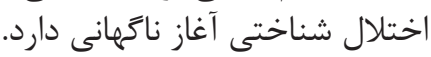

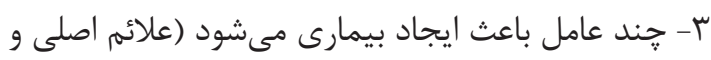

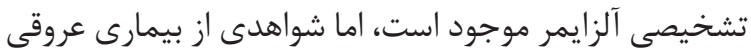

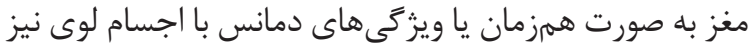

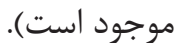

F أ- مدارك و شواهدى از بيمارى ديخرى، مصرف دارو يا

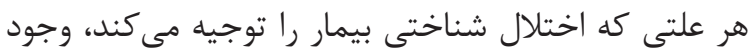

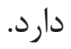

\section{ج- AD محتمل با توجه به شواهد ياتوفيزيولوزيك د- AD ممكن با توجه به شواهد ياتوفيزيولوزيك}

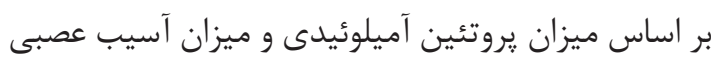
تعريف مىشود ( (r، r، ().)

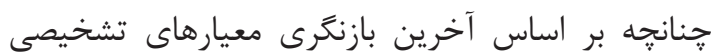

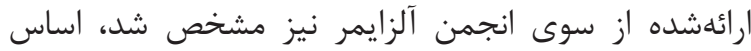

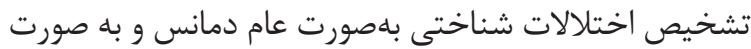

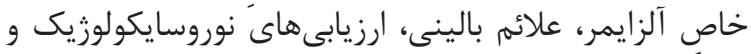

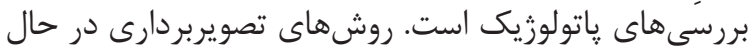

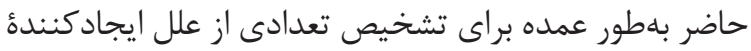

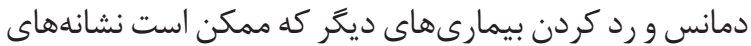

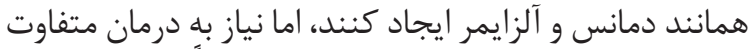

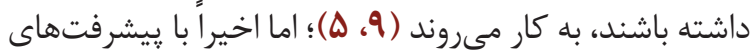

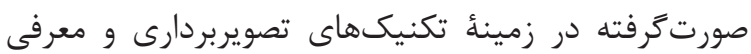

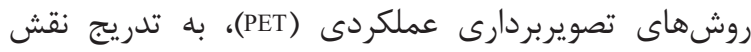

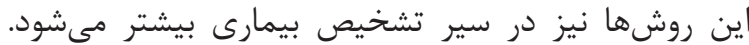

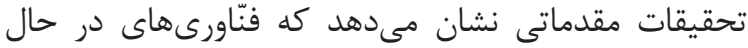

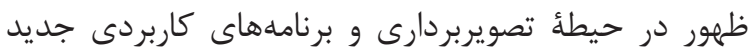

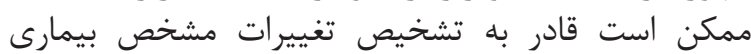

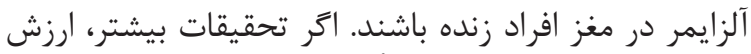

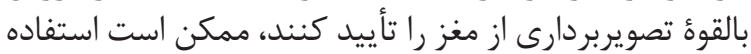

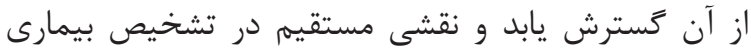

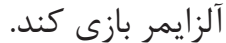

\section{بحث ويافته ها}

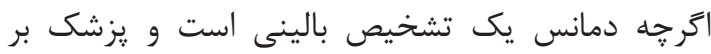

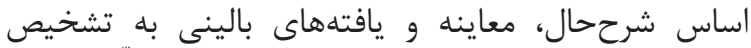

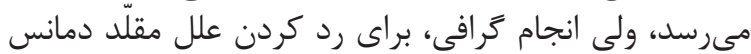

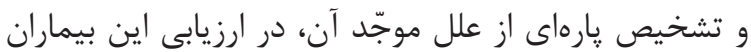

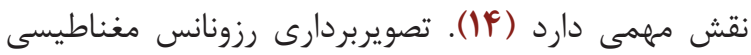


بو - تغييرات مشاهدهشده در MRI: آر جه CT اسكن

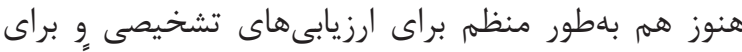

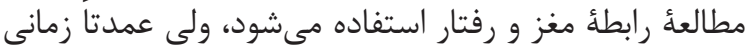

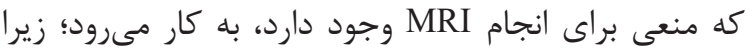

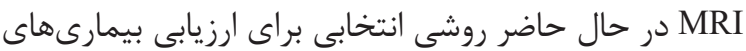

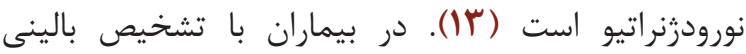

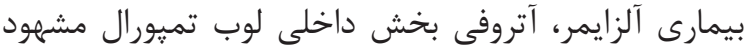

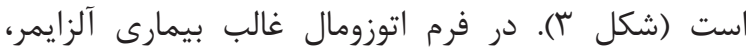

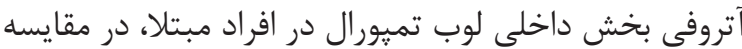

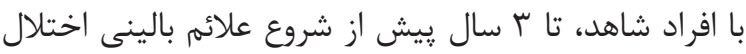

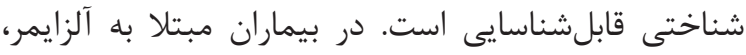

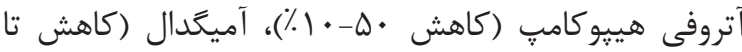

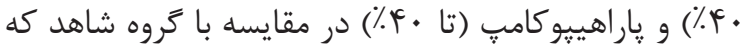

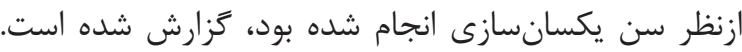

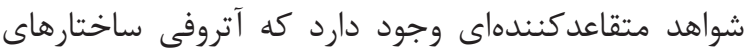

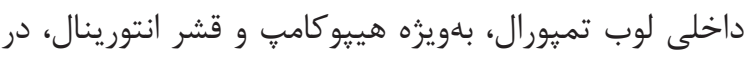

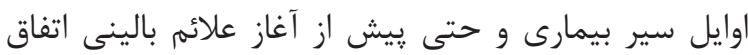

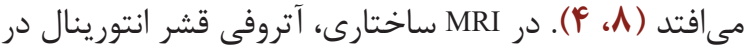

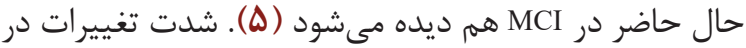

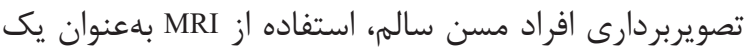

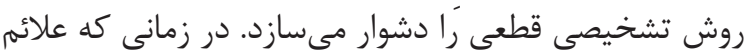

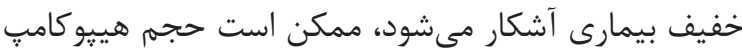

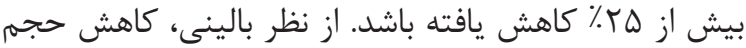

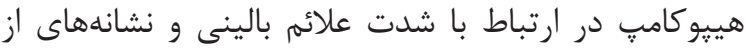

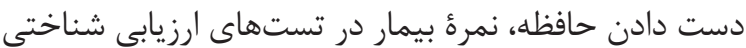

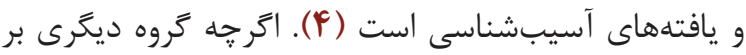

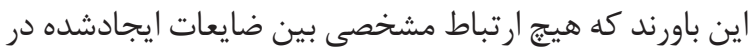

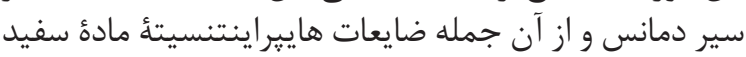

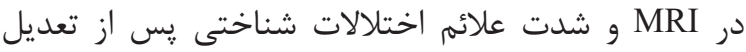

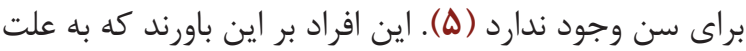

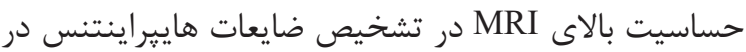

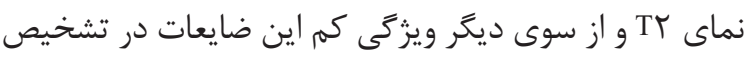

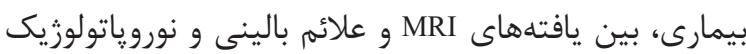
ارتباط ضعيفى برقرار است (9) بافتهاي (9). در پارهاى از انواع دمانس، تغييرات مشخصى در
روانشناختى تطابقى ندارد. در بيماران مبتلا به آلزايمر ميزان

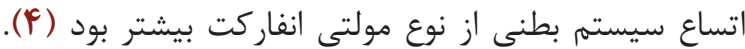

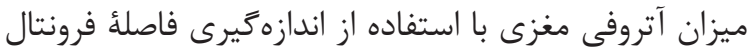

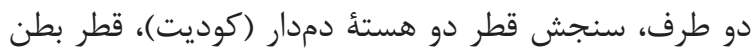

سوم و بطنهاى جانبى تعريف مى شود (ه) دمار (ك).

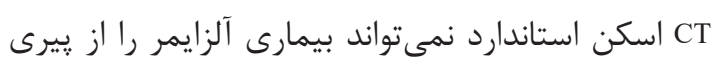

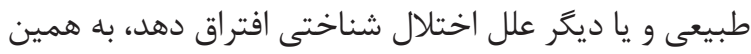

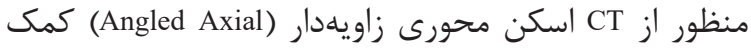

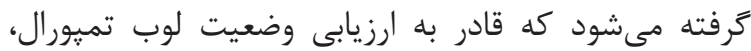

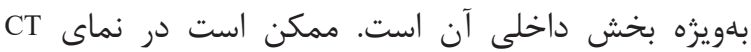

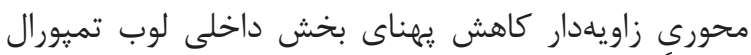

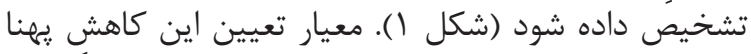

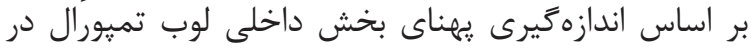

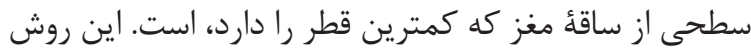

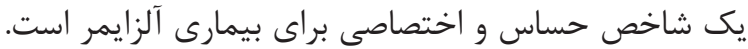

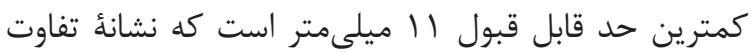

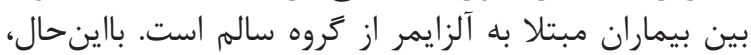

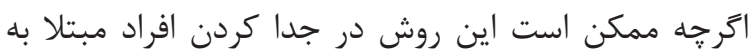

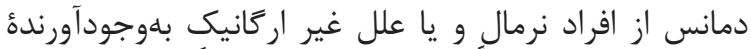

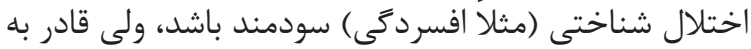

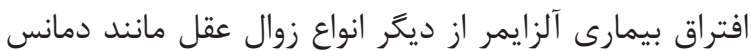

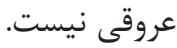

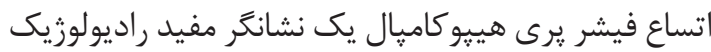

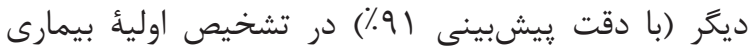

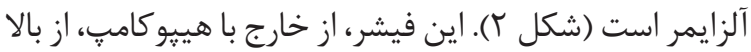

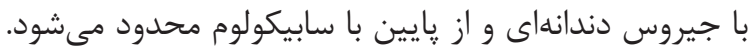

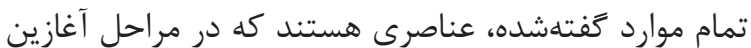

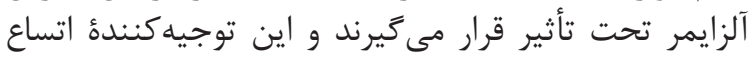

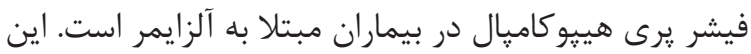

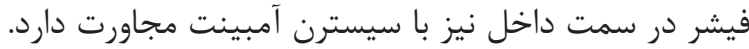

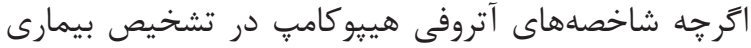

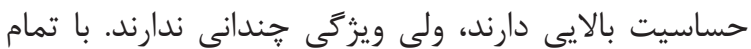

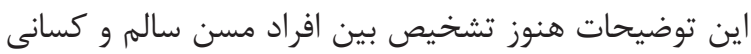

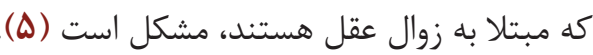
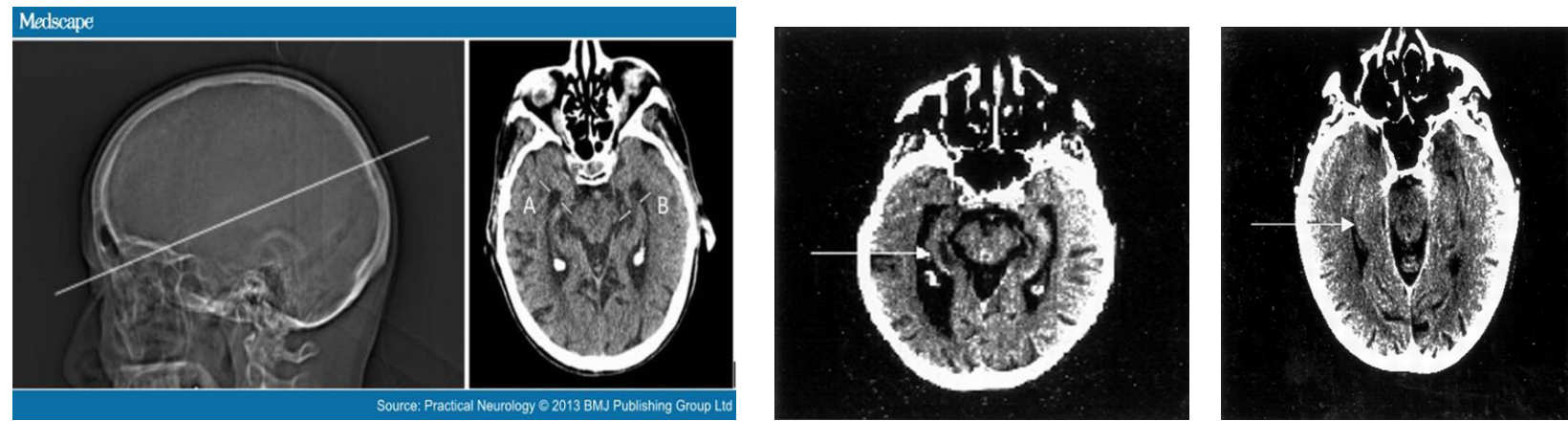

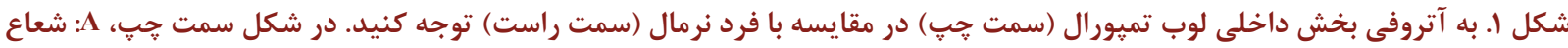

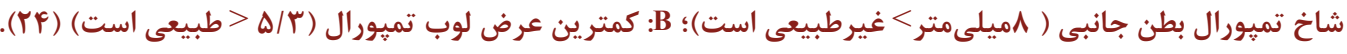




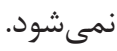

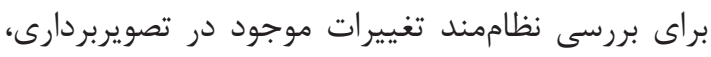

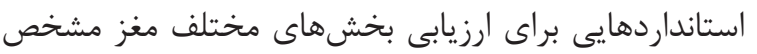

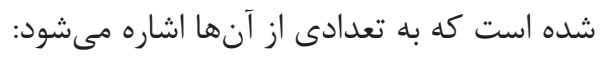

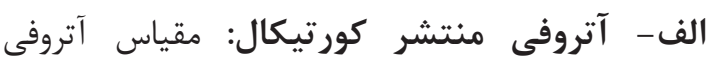

كورتيكال توسط Pasquier و همكارانش ابداع شده است (II).

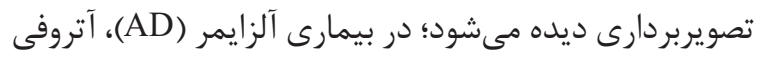

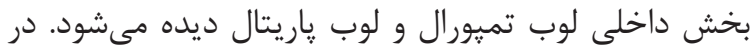

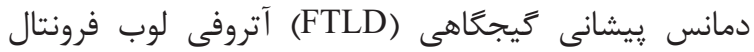

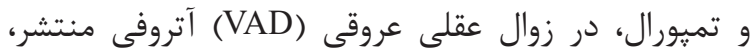

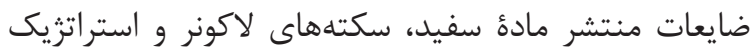

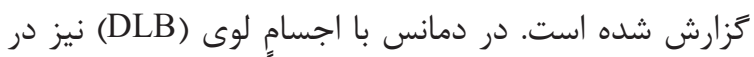
مقايسه با انواع ديكر زوال عقل، معمولا اختلال خاصى دئ ديده
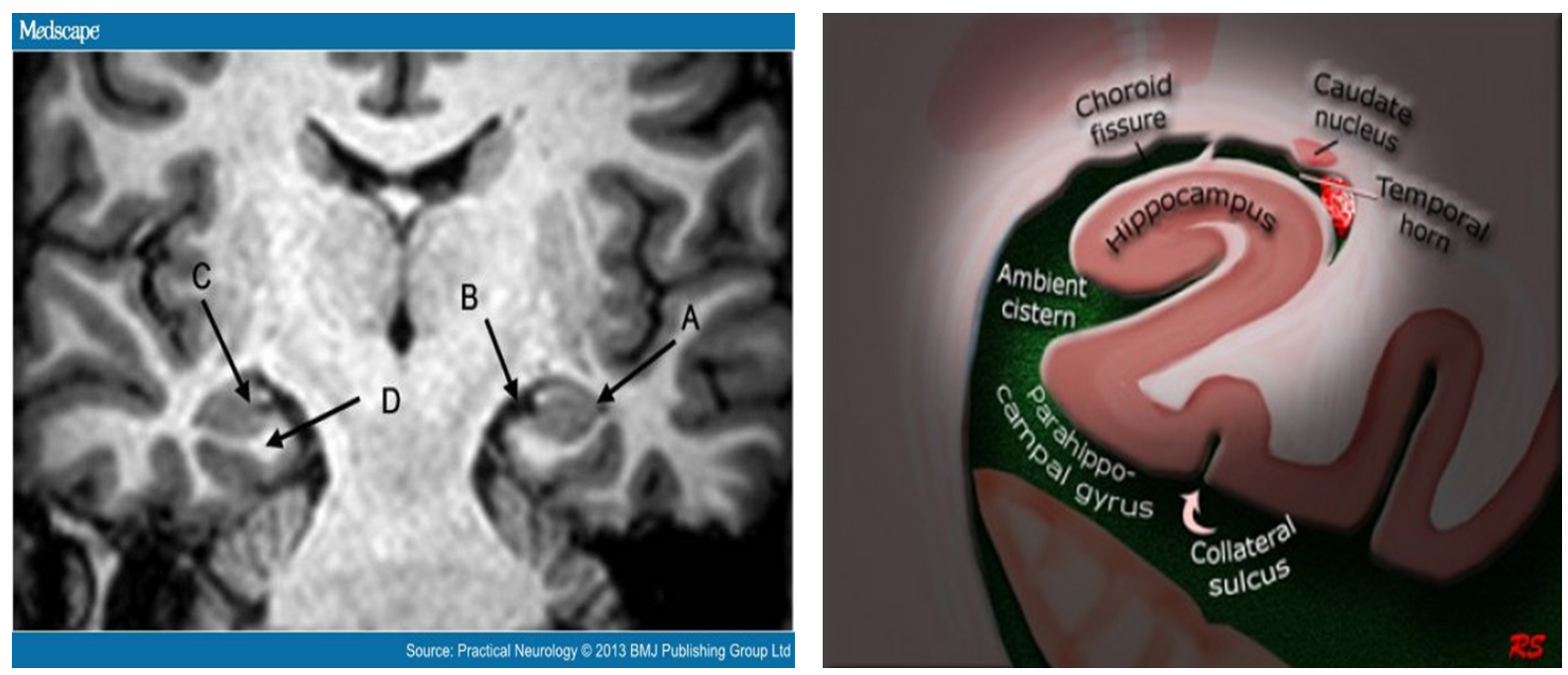

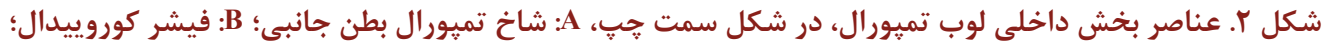

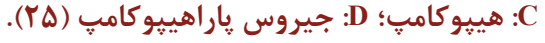

است. نمرهٔ بالا در اين روش براى تشخيص بيمارى آلزايمر

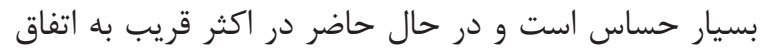

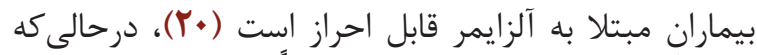

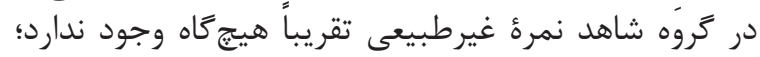

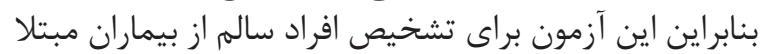

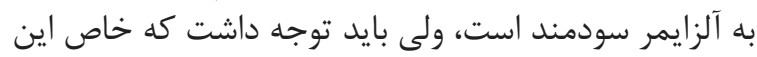

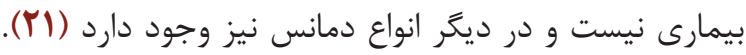

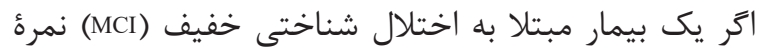

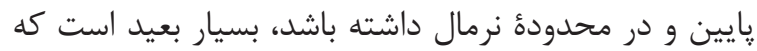

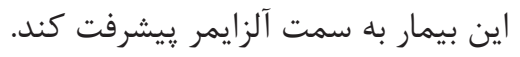

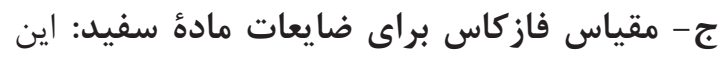

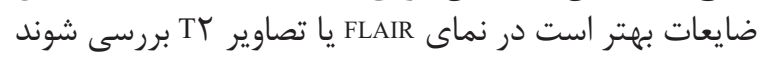

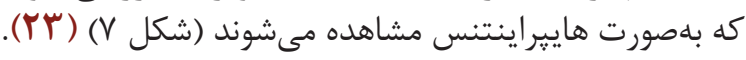
فازكاس •: عدم وجود ضايعه و يا يك ضايعه فاز كاس ا: ضايعات نقطهنقطئ جند

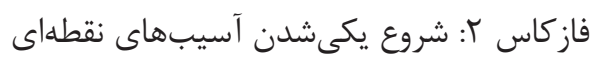

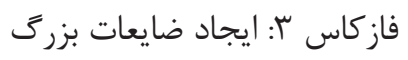

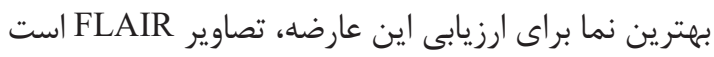

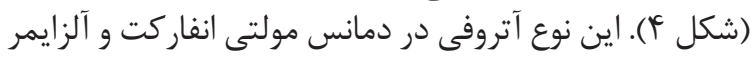

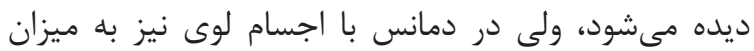

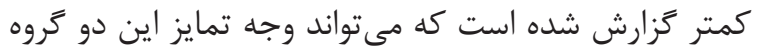

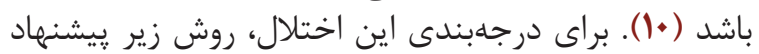

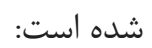

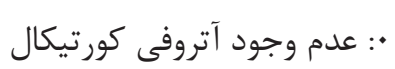
1: آتروفى خفيف: باز شدن سولكوس آنها

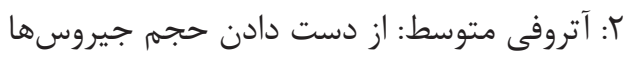

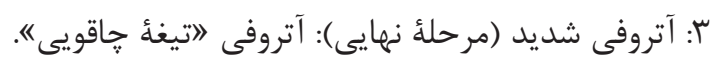

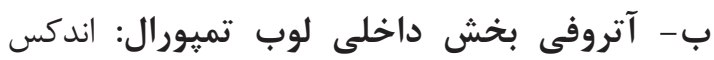

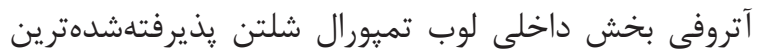

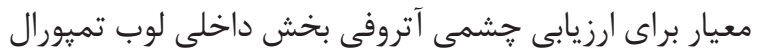

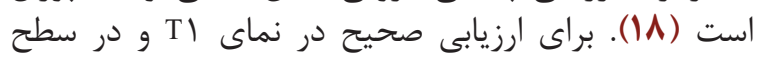

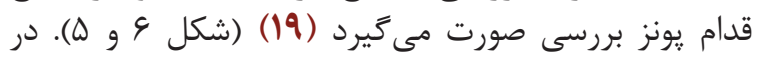

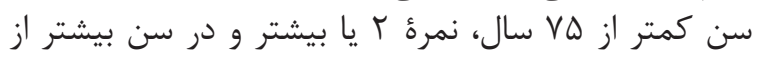

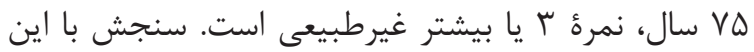

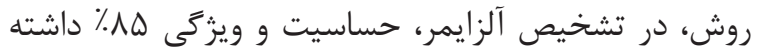



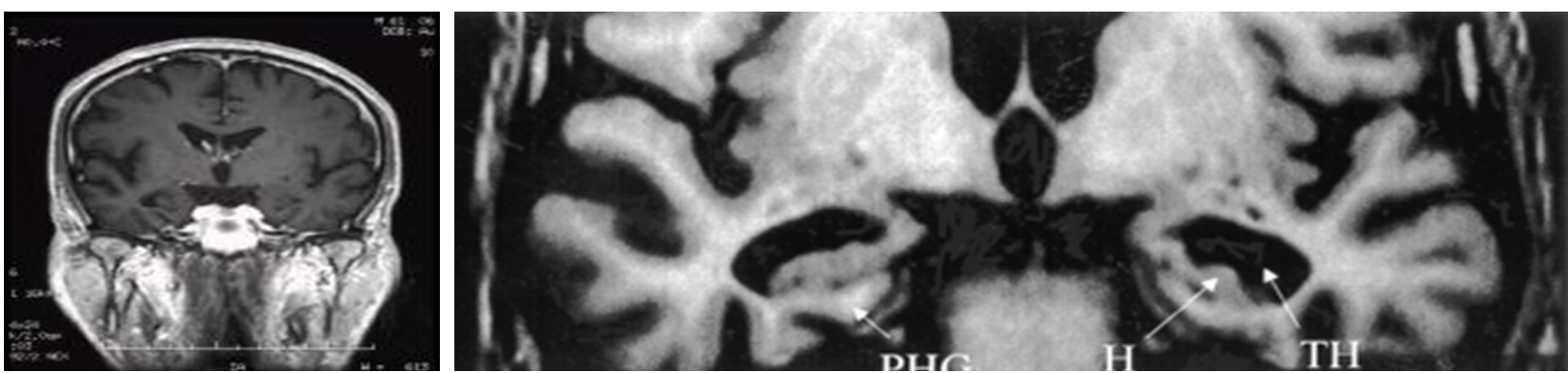

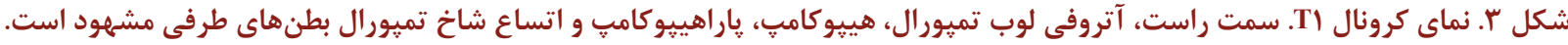

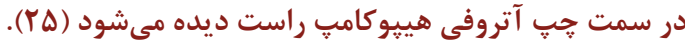

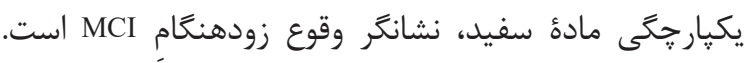

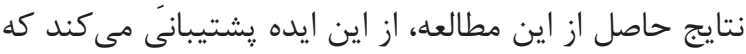

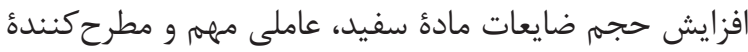

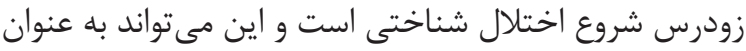

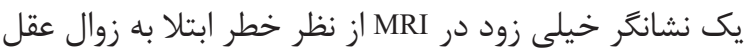

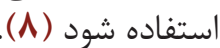

د- بررسى از نظر وجود سكتهُ مغزى استراتزيك:

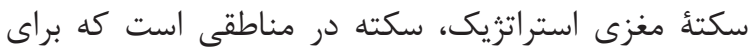

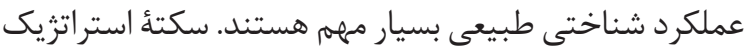

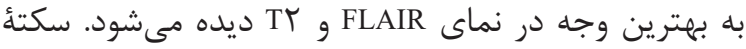
تالاموس دوطرفه اغلب با اختلال در عملكرد مغز ارتباط دارد دارد

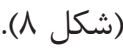

فاز كاس ال در افراد مسن طبيعى در نظر كَرفته مىشود و

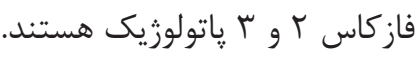

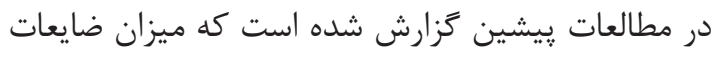

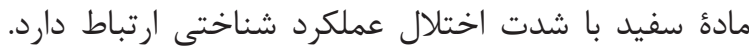

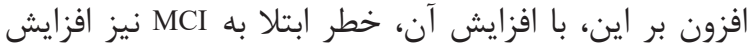

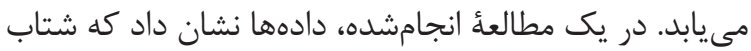

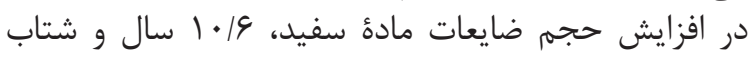

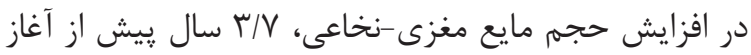

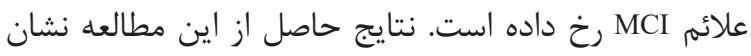

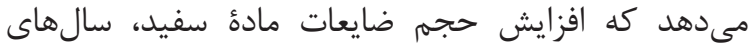

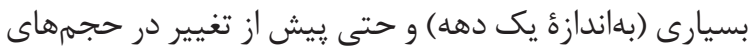

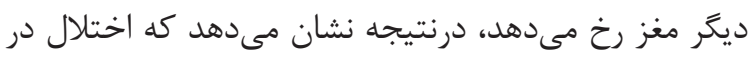

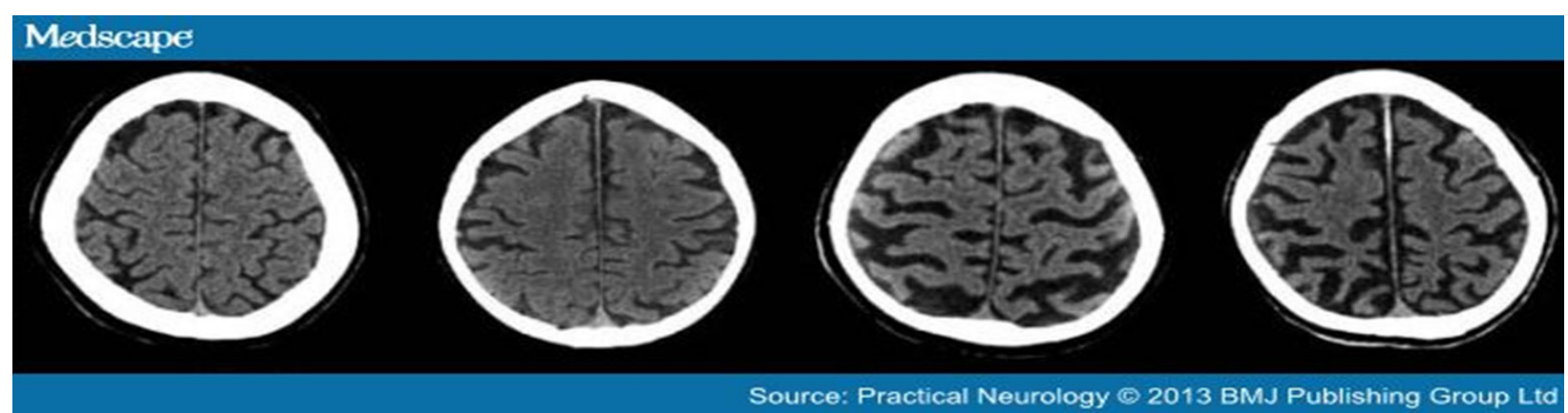

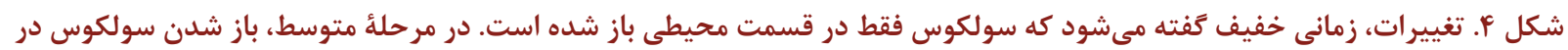

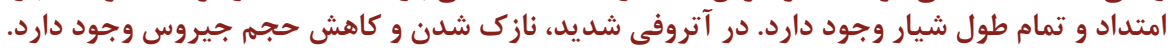

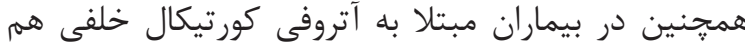

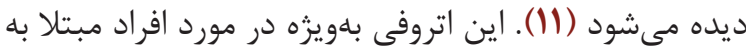

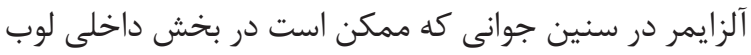

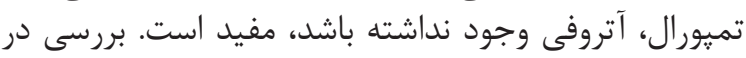

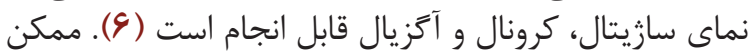

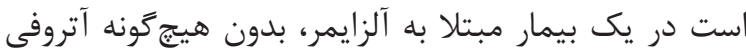

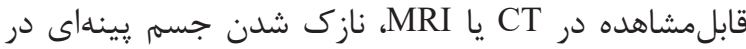

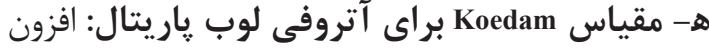

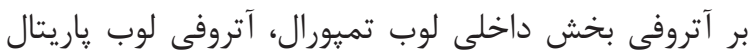

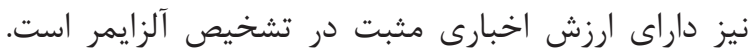

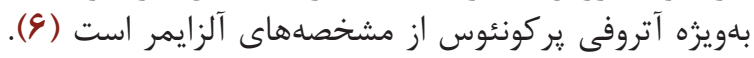

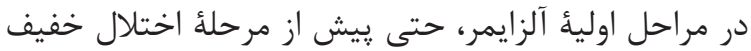

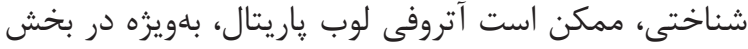

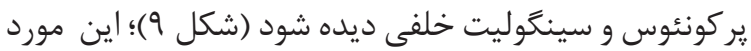




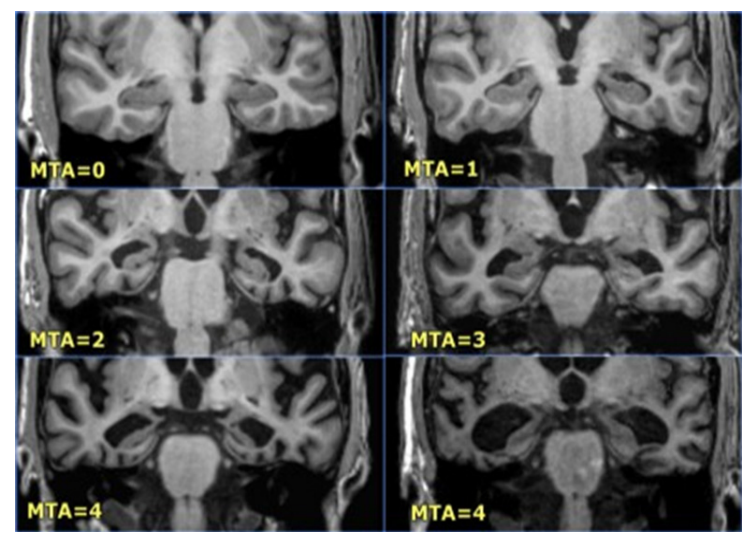

شكل \&. آتروفى عناصر داخلى لوب تميورال (צ؟)

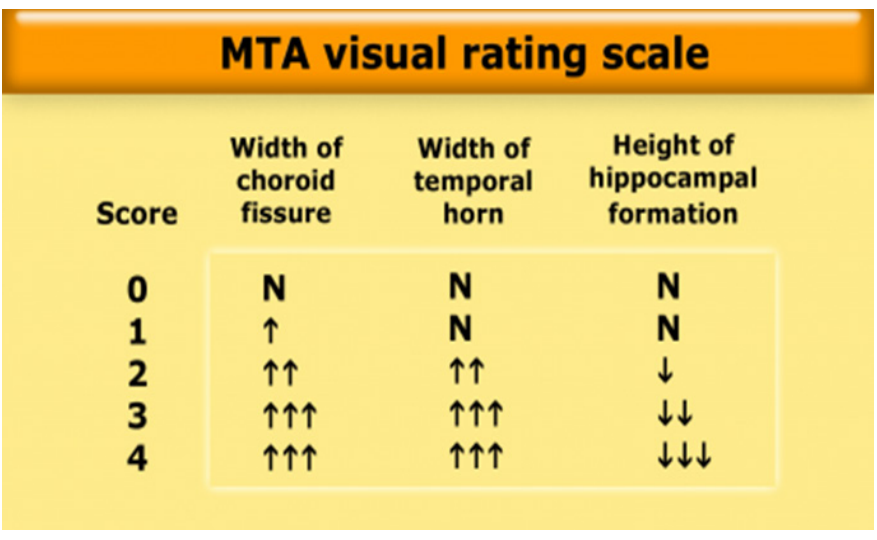

شكل ه. درجهبندى آتروفى بخش داخلى لوب تميورال
است. از سوى ديخر بيشرفتهاى صورت گرفته در روشهاى

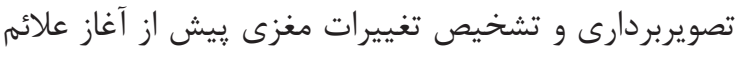

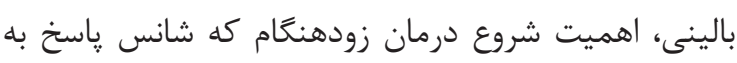

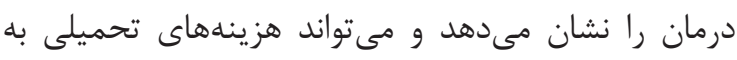

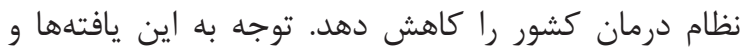
شناخت آنها در تشخيص بيماران حائز اهميت است (1I).

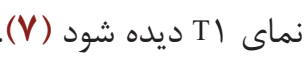

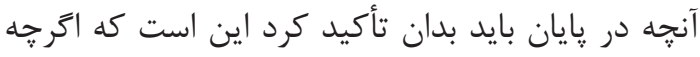

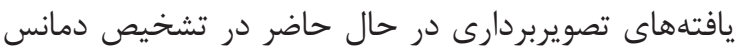

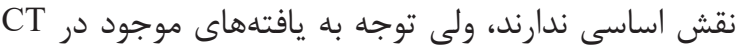

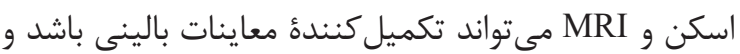

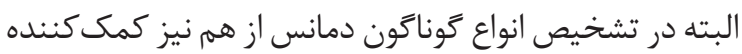

\begin{tabular}{|ll}
\hline \multicolumn{2}{c}{ Strategic Infarctions } \\
\hline Med Cerebral Artery & $\begin{array}{l}\text { Parieto-temporal or } \\
\text { temp-occip association areas } \\
\text { Angular Gyrus }\end{array}$ \\
Post Cerebral Artery & $\begin{array}{l}\text { Paramedian thalamic, } \\
\text { inferior medial temporal lobe }\end{array}$ \\
Watershed infarctions & $\begin{array}{l}\text { Superior frontal or parietal } \\
\text { lacunar infactions }\end{array}$ \\
\hline
\end{tabular}

شكل ^. مناطق وقوع سكتهُ مغزى استراتزيك

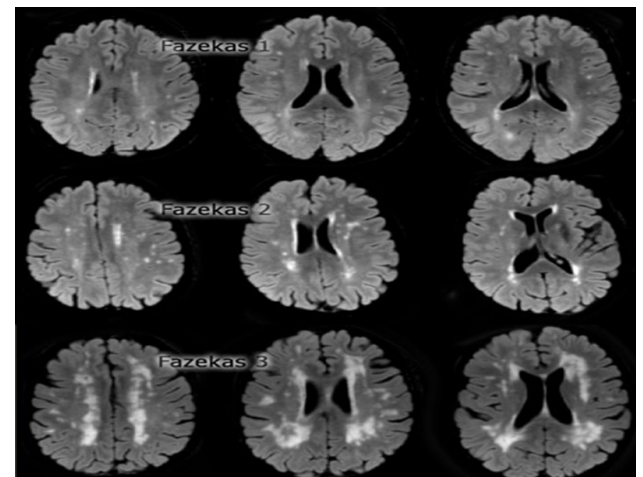

شكل V. ضايعات ماده سفيد (r^)

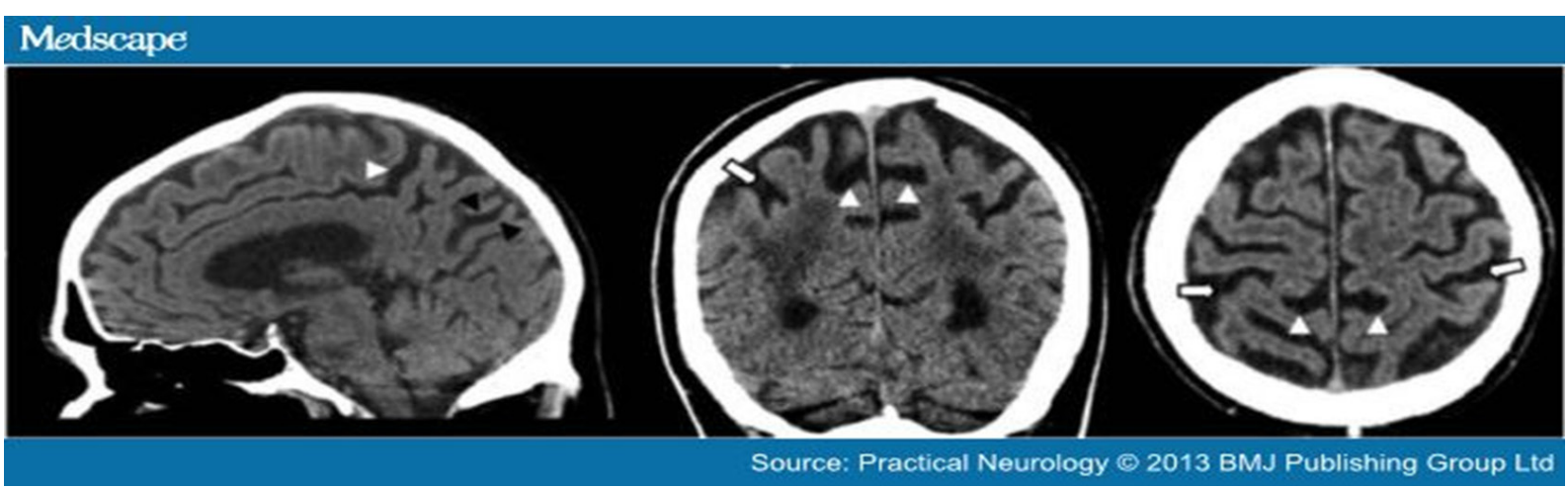

شكل 9. آتروفى لوب هاريتال، سر فلش سياه: باز شدن سولكوس پاريتواكسيبيتال؛ سر فلش سفيد: باز شدن سولكوس سينگوليت خلفى؛

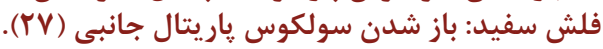




\section{References}

1. Albert MS, DeKosky ST, Dickson D, Dubois B, Feldman HH, Fox NC et al. The diagnosis of mild cognitive impairment due to Alzheimer's disease: recommendations from the National Institute on Aging-Alzheimer's Association workgroups on diagnostic guidelines for Alzheimer's disease. Alzheimers Dement. 2011 May;7(3):270-9. https://doi.org/10.1016/j. jalz.2011.03.008 PMID:21514249

2. Sperling RA, Aisen PS, Beckett LA, Bennett DA, Craft $\mathrm{S}$, Fagan AM et al. Toward defining the preclinical stages of Alzheimer's disease: recommendations from the National Institute on Aging-Alzheimer's Association workgroups on diagnostic guidelines for Alzheimer's disease. Alzheimers Dement. 2011 May;7(3):280 92. $\quad$ https://doi.org/10.1016/j.jalz.2011.03.003 PMID:21514248

3. Jack CR Jr, Albert MS, Knopman DS, McKhann GM, Sperling RA, Carrillo MC et al. Introduction to the recommendations from the National Institute on Aging-Alzheimer's Association workgroups on diagnostic guidelines for Alzheimer's disease. Alzheimers Dement. 2011 May;7(3):257-62. https://doi. org/10.1016/j.jalz.2011.03.004 PMID:21514247

4. Soininen H, Puranen M, Riekkinen PJ. Computed tomography findings in senile dementia and normal aging. J Neurol Neurosurg Psychiatry. 1982 Jan;45(1):50 4. https://doi.org/10.1136/jnnp.45.1.50 PMID:7062070

5. Tarakad S. Ramachandran, Alzheimer Disease Imaging. Updated: Mar 27, 2014. Available from URL: $\underline{\text { ttp:// }}$ www.emedicine.medscape.com/article/336281

6. Frederik Barkhof, Marieke Hazewinkel, Maja Binnewijzend and Robin Smithuis. Dementia: role of MRI. Radiology Assistant. Publicationdate January 9, 2012.

7. Knopman DS, DeKosky ST, Cummings JL, Chui H, Corey-Bloom J, Relkin N et al. Practice parameter: diagnosis of dementia (an evidence-based review). Report of the Quality Standards Subcommittee of the American Academy of Neurology. Neurology. 2001 May;56(9):1143-53. https://doi.org/10.1212/ WNL.56.9.1143 PMID:11342678

8. Whitwell JL, Jack CR Jr. Neuroimaging in dementia. Neurol Clin. 2007 Aug;25(3):843-57. https://doi. org/10.1016/j.ncl.2007.03.003 PMID:17659193

9. Pantano P, Caramia F, Pierallini A. The role of MRI in dementia. Ital J Neurol Sci. 1999;20(5 Suppl):S250-3. $\quad$ https://doi.org/10.1007/s100729970006 PMID: 10662960

10. Whitwell JL, Weigand SD, Shiung MM, Boeve BF, Ferman TJ, Smith GE et al. Focal atrophy in dementia with Lewy bodies on MRI: a distinct pattern from Alzheimer's disease. Brain. 2007 Mar;130(Pt 3):708-19. https://doi.org/10.1093/brain/awl388 PMID:17267521

11. Mortimer AM, Likeman M, Lewis TT. Neuroimaging in dementia: a practical guide. Pract Neurol. 2013 Apr;13(2):92-103. https://doi.org/10.1136/practneurol-2012-000337 PMID:23468560

12. Vitali P, Migliaccio R, Agosta F, Rosen HJ, Geschwind MD. Neuroimaging in dementia. Semin Neurol. 2008 Sep;28(4):467-83. https://doi. org $/ 10.1055 / \mathrm{s}-0028-1083695$

PMID: 18843575
13. Tartaglia MC, Rosen HJ, Miller BL. Neuroimaging in dementia. Neurotherapeutics. 2011 Jan;8(1):8292. $\quad$ https://doi.org/10.1007/s13311-010-0012-2 PMID:21274688

14. Guy M. Mc.Khann, David S. Knopman, Howard Chertkow, Bradley T. Hyman, Clifford R. Jack, Jr., Claudia H. Kawas et al. The diagnosis of dementia due to Alzheimer's disease: Recommendations from the National Institute on Aging and the Alzheimer's Association workgroup. Alzheimers Dement. 2011 May;7(3):263-9

15. Dementia UK. Alzheimer's Society: http://alzheimers. org.uk (accessed March 2012).

16. McDonald CR, McEvoy LK, Gharapetian L, Fennema-Notestine C, Hagler DJ Jr, Holland D et al.; Alzheimer's Disease Neuroimaging Initiative. Regional rates of neocortical atrophy from normal aging to early Alzheimer disease. Neurology. 2009 Aug;73(6):45765. https://doi.org/10.1212/WNL.0b013e3181b16431 PMID:19667321

17. Whitwell JL, Przybelski SA, Weigand SD, Knopman DS, Boeve BF, Petersen RC et al. 3D maps from multiple MRI illustrate changing atrophy patterns as subjects progress from mild cognitive impairment to Alzheimer's disease. Brain. 2007 Jul;130(Pt 7):1777-86. https://doi.org/10.1093/brain/awm112 PMID: 17533169

18. Scheltens P, Leys D, Barkhof F, Huglo D, Weinstein $\mathrm{HC}$, Vermersch P et al. Atrophy of medial temporal lobes on MRI in "probable" Alzheimer's disease and normal ageing: diagnostic value and neuropsychological correlates. J Neurol Neurosurg Psychiatry. 1992 Oct;55(10):967-72. https://doi.org/10.1136/ jnnp.55.10.967 PMID:1431963

19. Wattjes MP, Henneman WJ, van der Flier WM, de Vries O, Träber F, Geurts JJ et al. Diagnostic imaging of patients in a memory clinic: comparison of MR imaging and 64-detector row CT. Radiology. 2009 Oct;253(1):174-83. https://doi.org/10.1148/radiol.2531082262 PMID:19635835

20. Burton EJ, Barber R, Mukaetova-Ladinska EB, Robson J, Perry RH, Jaros E et al. Medial temporal lobe atrophy on MRI differentiates Alzheimer's disease from dementia with Lewy bodies and vascular cognitive impairment: a prospective study with pathological verification of diagnosis. Brain. 2009 Jan;132(Pt 1):195-203. https://doi.org/10.1093/brain/awn298 PMID: 19022858

21. van de Pol LA, Hensel A, van der Flier WM, Visser PJ, Pijnenburg YA, Barkhof $F$ et al. Hippocampal atrophy on MRI in frontotemporal lobar degeneration and Alzheimer's disease. J Neurol Neurosurg Psychiatry. 2006 Apr;77(4):439-42. https://doi.org/10.1136/ jnnp.2005.075341 PMID:16306153

22. Cohn-Hokke PE, Elting MW, Pijnenburg YA, van Swieten JC. Genetics of dementia: update and guidelines for the clinician. Am J Med Genet B Neuropsychiatr Genet. 2012 Sep;159B(6):628-43. https://doi. org/10.1002/ajmg.b.32080 PMID:22815225

23. Wahlund LO, Barkhof F, Fazekas F, Bronge L, Augustin M, Sjögren M et al.; European Task Force on Age-Related White Matter Changes. A new rating scale for age-related white matter changes applicable to MRI 
and CT. Stroke. 2001 Jun;32(6):1318-22. https://doi. org/10.1161/01.STR.32.6.1318 PMID:11387493

24. Frisoni GB, Beltramello A, Weiss C, Geroldi C, Bianchetti A, Trabucchi M. Linear measures of atrophy in mild Alzheimer disease. AJNR Am J Neuroradiol. 1996 May;17(5):913-23. PMID:8733967

25. Chaim TM, Duran FL, Uchida RR, Périco CA, de Castro CC, Busatto GF. Volumetric reduction of the corpus callosum in Alzheimer's disease in vivo as assessed with voxel-based morphometry. Psychiatry Res. 2007 Jan;154(1):59-68. https://doi.org/10.1016/j. pscychresns.2006.04.003

PMID:17174533
26. Jobst KA, Smith AD, Szatmari M, Molyneux A, Esiri ME, King E et al. Detection in life of confirmed Alzheimer's disease using a simple measurement of medial temporal lobe atrophy by computed tomography. Lancet. 1992 Nov;340(8829):1179-83. https://doi. org/10.1016/0140-6736(92)92890-R PMID:1359259

27. Harper L, Barkhof F, Scheltens P, Schott JM, Fox NC. An algorithmic approach to structural imaging in dementia. J Neurol Neurosurg Psychiatry. 2014 Jun;85(6):692-8. https://doi.org/10.1136/jnnp-2013306285 PMID:24133287

28. Fazekas F, Chawluk JB, Alavi A, Hurtig HI, Zimmerman RA. MR signal abnormalities at $1.5 \mathrm{~T}$ in Alzheimer's dementia and normal aging. AJR Am J Roentgenol. 1987 Aug;149(2):351-6. https://doi.org/10.2214/ ajr.149.2.351 PMID:3496763 